\title{
Present status of undoped semi-insulating LEC bulk GaAs as a radiation spectrometer
}

\author{
Douglas S. McGregor ${ }^{\text {a,* }}$, Ronald A. Rojeski ${ }^{\text {a }}$, Glenn F. Knoll ${ }^{\text {a }}$, Fred L. Terry, Jr. ${ }^{\text {, }}$ \\ Jack East $^{\mathrm{b}}$, Yosef Eisen ${ }^{\mathrm{c}}$ \\ ${ }^{a}$ Department of Nuclear Engineering, Unversity of Michigan, Ann Arbor, MI 48109, USA \\ ${ }^{b}$ Department of Electrical Engineering and Computer Science, University of Michtgan, Ann Arbor, MI 48109, USA \\ " Soreq Nuclear Research Center, Israel Atomic Energy Commission, Yaune 70600, Israel
}

(Received 17 September 1993; revised form received 17 December 1993)

Bulk GaAs has undergone extensive research by several groups in order to ascertain its usefulness as a room temperature radiation spectrometer. The results of an experimental program studying the properties of detectors fabricated from bulk GaAs are summarized in this paper. Electric field models of the active region are compared with measured results. Limitations of bulk LEC GaAs as a material for radiation spectrometers are discussed.

\section{Introduction}

In general, the requirement for room temperature operation of a semiconducting material for radiation detection is a relatively large band gap energy such that thermal generation of signal carriers is kept to a minimum. Conversely, the requirement for high resolution spectroscopy demands that the band gap be small enough to allow for the production of a large number of charge carriers per ionizing event. Therefore, a compromise is necessary if a material is to be considered as a room temperature operated semiconductor radiation spectrometer. Early studies indicated that the optimum band gap should be near 1.4 to $1.5 \mathrm{eV}[1,2]$. The material should also have a relatively high $Z$ value if applied in gamma ray spectroscopy. High carrier mobilities and long carrier lifetimes are also needed to ensure efficient charge carrier extraction to reduce position dependent charge collection effects [3].

Gallium arsenide (GaAs) offers an attractive choice for such a detector and has been studied by a number of different groups over the past decades [4-22]. The material is a III-V compound semiconductor with atomic numbers of $31 / 33$ and a density of $5.32 \mathrm{~g} / \mathrm{cm}^{3}$. The band gap energy is $1.42 \mathrm{eV}$ at room temperature and the average energy required to produce an electron-hole pair is approximately $4.2 \mathrm{eV}$. The Fano factor has been quoted to be near 0.18 , indicating that

* Corresponding author. acceptably high resolution can be achieved [7,23]. The electron mobility at low electric fields can exceed 8000 $\mathrm{cm}^{2} / \mathrm{V}$ s. At fields above $3 \times 10^{3} \mathrm{~V} / \mathrm{cm}$, the electron mobility decreases due to the intervalley exchange of electrons from the direct conduction band valley ( $\Gamma$ valley) to a lower mobility valley ( $\mathrm{L}$ valley) $[24,25]$. In contrast, holes have mobilities near $400 \mathrm{~cm}^{2} / \mathrm{V} \mathrm{s}$. Both electrons and holes have saturated velocities near $10^{7}$ $\mathrm{cm} / \mathrm{s}$, however holes require higher fields than electrons to reach saturated velocity. The intrinsic carrier concentration of GaAs is $1.79 \times 10^{6}$ per $\mathrm{cm}^{3}$ which corresponds to a resistivity of $10^{8} \Omega \mathrm{cm}$ [24], indicating that thermally produced generation current should be relatively low at room temperature. Theory predicts that carrier lifetimes in a very pure and well ordered crystal should approach $0.1 \mathrm{~ms}[7,26]$. However such long lifetimes are not observed in practice and carrier lifetimes are generally measured to be on the order of only a few nanoseconds.

GaAs detectors have been studied by a number of research groups over the past 25 years. Detectors fabricated from liquid phase epitaxially (LPE) grown high purity GaAs were the first compound semiconductor spectrometers that demonstrated acceptable energy resolution for gamma rays [6,7]. The LPE layers were grown on n-type $\langle 100\rangle$ oriented GaAs substrates and ranged from 60 to $80 \mu \mathrm{m}$ thick after processing. The basic device structure consisted of a Au Schottky contact and an alloyed $\mathrm{Ga}-\mathrm{In}$ ohmic contact. Measurements were reported with $2-3 \%$ FWHM energy resolution for $122 \mathrm{keV}$ gamma rays at room temperature. Similar LPE GaAs detectors were reported to have 
epitaxial thicknesses ranging from 30 to $120 \mu \mathrm{m}$ with Schottky contacts fabricated from either Au or Pd $[8,10]$. Room temperature spectroscopic results were reported with $0.3 \%$ energy resolution for $5.5 \mathrm{MeV}$ alpha particles from ${ }^{241} \mathrm{Am}$ and better than $5 \%$ energy resolution for $60 \mathrm{keV}$ gamma rays from ${ }^{241} \mathrm{Am}$. Vapor phase epitaxy (VPE) and other LPE GaAs Schottky contact detectors were reported with similar results [9]. The thicknesses of the VPE layers were only $8 \mu \mathrm{m}$ and material quality was reported to be inferior to that of LPE. However, the VPE layer detectors were measured to have $6 \%$ energy resolution for $41.5 \mathrm{keV} \mathrm{X}$-rays at room temperature. In all cases the VPE and LPE material was limited to thicknesses near $100 \mu \mathrm{m}$ or less and proved difficult to reproduce. The thin layers of LPE material were extremely inefficient for high energy gamma ray detectors and utilization was restricted to spectroscopic measurements of charged particles, low energy gamma rays, and X-rays. Later attempts were made to improve gamma ray detection efficiency by growing $\mathrm{Cr}$ doped LPE layers on each side of a $\mathbf{n}^{+}$ GaAs substrate [13]. The counting rate was reported to improve $20 \%$ when both detectors were operated in common mode as compared to one detector operated in single mode.

Epitaxial detectors have very low gamma ray detection efficiency and do not offer a satisfactory alternative to $\mathrm{Ge}$ or $\mathrm{Si}$ based detectors. Detectors made from LPE or VPE GaAs material are expensive and difficult to reproduce. Bulk GaAs material of 20 years ago was inferior in quality and inadequate for use as a radiation spectrometer. Due to material problems, research concerning $\mathrm{GaAs}$ as a possible detector material was discontinued in the late 1970s and early 1980s.

Since then, GaAs has undergone extensive research as a material for VLSI applications as well as the base substrate for several exotic electronic devices. Demand in the electronics industry motivated extensive research to improve the material quality of bulk GaAs. GaAs is now commonly used for high speed transistors, quantum well diodes, laser diodes, and microwave oscillators. Due to the demand for high quality GaAs, high purity bulk GaAs is now readily available from a number of commercial vendors. Thus, GaAs has undergone renewed interest as a possible choice for detection of ionizing radiation. Bulk GaAs appears to be attractive as a room temperature operated gamma ray and alpha particle spectrometer [17-20], as a detector of minimum ionizing particles [14], and as a possible detector material for dark matter searches [21]. High quality GaAs in bulk form offers a promising medium from which thick room temperature operated radiation spectrometers might be fabricated. This paper describes the present status of undoped semi-insulating (SI) liquid encapsulated Czochralski (LEC) bulk GaAs as a material for radiation spectroscopy.

\section{Detectors fabricated from bulk GaAs}

Bulk GaAs was investigated as a possible room temperature conductivity detector as early as 1960 with reported sample dimensions of $1 \mathrm{~cm} \times 0.2 \mathrm{~cm} \times 0.1 \mathrm{~cm}$ [4]. Bulk GaAs conductivity counters consisted primarily of ohmic contacts on opposite sides of a bulk crystal, and the detectors often demonstrated undesirable low frequency current oscillations at electric fields above $300 \mathrm{~V} / \mathrm{cm}$ [27-33]. Early Schottky contact based bulk $\mathrm{GaAs}$ detector structures consisted of a $\mathrm{Au}$ Schottky contact and a Sn ohmic contact [5]. The energy resolution for $5.15 \mathrm{MeV}$ alpha particles was reported to be $7.7 \%$, and the detectors did not suffer from current oscillations [5]. The poor energy resolution observed was attributed mainly to short carrier lifetimes.

Present day GaAs detectors fabricated from undoped SI LEC bulk GaAs have demonstrated improved alpha particle resolution over earlier bulk GaAs detectors. Schottky contact detectors have been reported to have energy resolution ranging from approximately 2 to $5 \%$ at FWHM for $5.5 \mathrm{MeV}$ alpha particles $[14,17,18]$. Alpha particle resolution was usually reported to be best when the detectors were irradiated from the front contact (Schottky contact) as opposed to the back contact (ohmic contact).

Figs. 1 and 2 show spectra taken in our laboratory with a $45 \mu \mathrm{m}$ thick bulk GaAs detector from a ${ }^{228} \mathrm{Th}$ source when irradiated from the front and back. The detector configuration was that as described in the literature [17]. The voltage appearing across the detector terminals was held at $55 \mathrm{~V}$, in which it was found that the active region extended completely across the device. The ${ }^{228} \mathrm{Th}$ source and daughter products emit alpha particles ranging in energy from 5.3 to $8.78 \mathrm{MeV}$. Alpha particles with energies of $5.5 \mathrm{MeV}$ and below

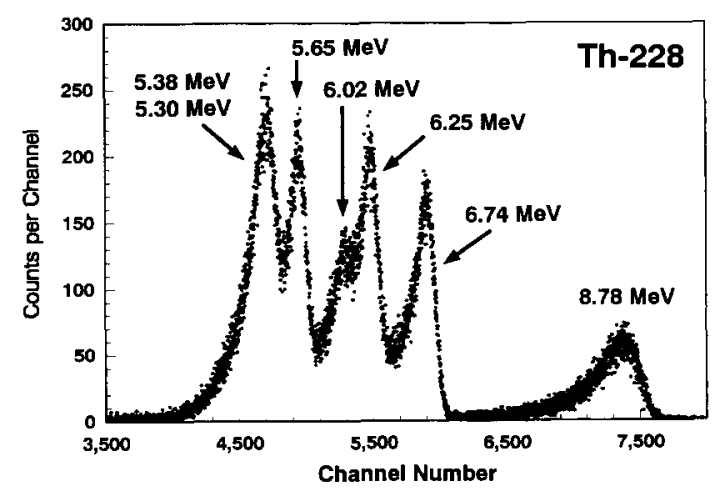

Fig. 1. Differential pulse height spectrum of Th-228 taken with a $45 \mu \mathrm{m}$ thick bulk GaAs detector. The detector was irradiated in vacuum from the front contact (Schottky contact). The energies indicated take into account energy loss of the alpha particles within the source. 


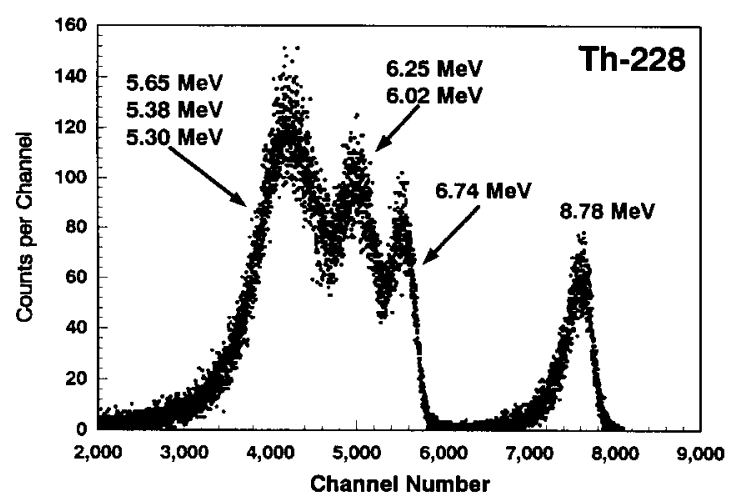

Fig. 2. Differential pulse height spectrum of Th-228 taken with a $45 \mu \mathrm{m}$ thick bulk GaAs detector. The detector was irradiated in vacuum from the back contact (ohmic contact). The energies indicated take into account energy loss of the alpha particles within the source.

have ranges of $20 \mu \mathrm{m}$ or less, therefore the highest density of charge is near the detector middle or the front contact when irradiated from the front. The electrons become the dominant carriers if irradiated from the front and the holes are dominant if irradiated from the back. From Figs. 1 and 2, it is obvious that the energy resolution for the lower energy alpha particles is much better for front irradiation than back irradiation. The range of the highest energy alpha particle emitted $(8.78 \mathrm{MeV})$ extends approximately 40 $\mu \mathrm{m}$ into the detector (Fig. 3) [34]. Hence, the highest density of charge is near the front of the detector when it is irradiated from the back. Since the $8.78 \mathrm{MeV}$ alpha particle produces more electron-hole pairs near the front of the detector when irradiated from the back, the electrons become the dominant signal carriers rather than the holes. From Figs. 1 and 2, the resolution of the $8.78 \mathrm{MeV}$ alpha particle improves

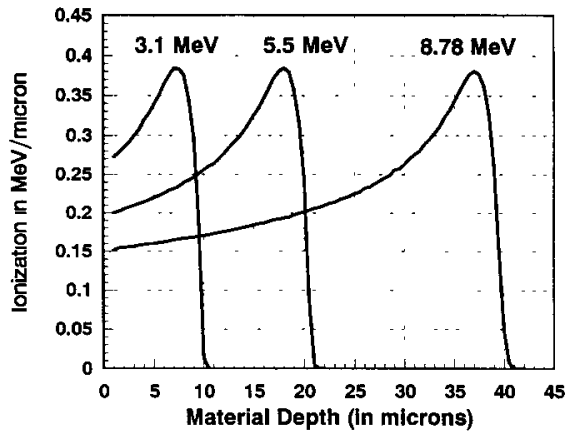

Fig. 3. Bragg ionization curves for $3.1,5.5$, and $8.78 \mathrm{MeV}$ alpha particles in GaAs.

slightly when irradiated from the back as opposed to the front. The spectra in Figs. 1 and 2 indicate that electrons are being collected more efficiently than holes.

In many cases, alpha particle pulses were not observed at all from back side irradiation, indicating that the detector active regions did not extend completely across the detectors. Although often used for semiconductor depletion depth characterization, modulated $\mathrm{C}-\mathrm{V}$ measurements on very high resistivity materials can often lead to erroneous conclusions. Unfortunately, the high resistivity of SI LEC GaAs creates a condition in which $C-V$ measurements at frequencies above $1 \mathrm{kHz}$ generally demonstrate no apparent change in capacitance, a result which can easily be misinterpreted as full extension of the active region across the diodes. A more detailed explanation of the difficulty can be found elsewhere [17,35]. As a result, different approaches were found to investigate the actual detector active region widths $[17,22]$. In our laboratory experiments, detectors were irradiated in vacuum with alpha particles of $3.1,5.5$, and $8.78 \mathrm{MeV}$ from the front
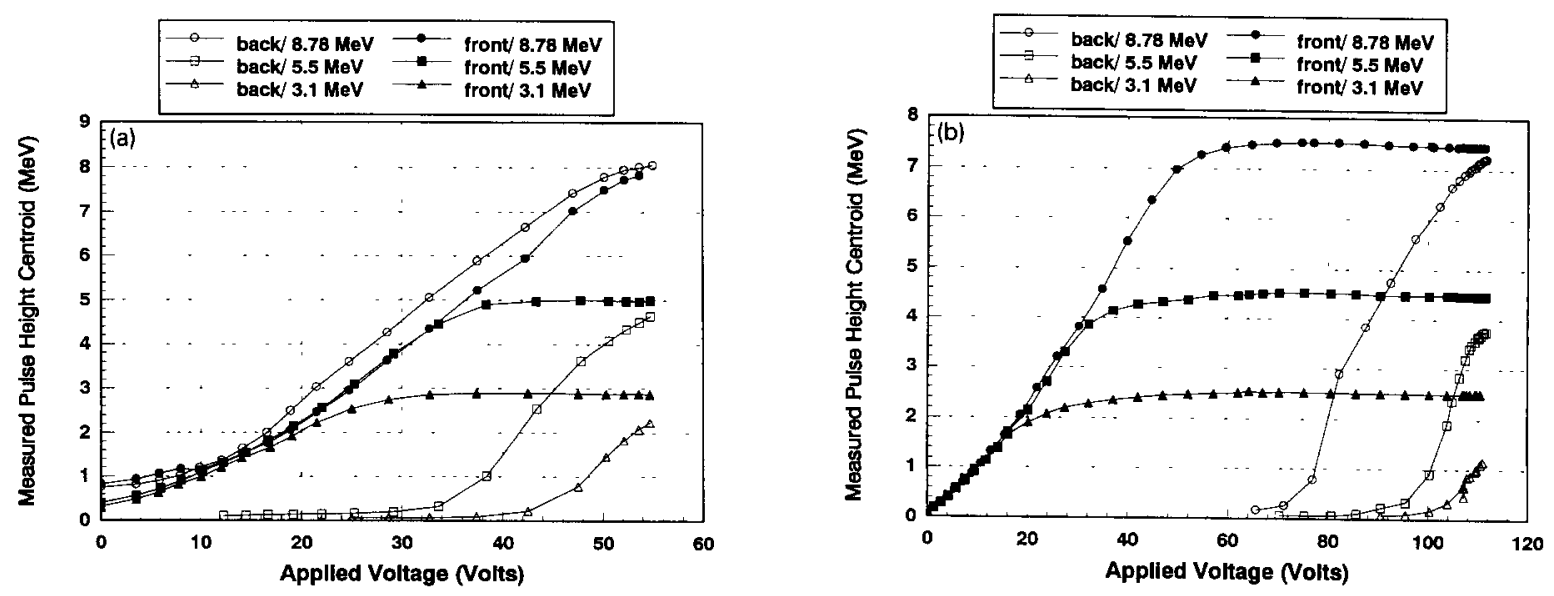

Fig. 4. Pulse height as a function of bias voltage for (a) a $45 \mu \mathrm{m}$ Schottky contact bulk GaAs detector and (b) a $100 \mu \mathrm{m}$ Schottky contact bulk GaAs detector. The detectors were irradiated in vacuum with $3.1,5.5$, and $8.78 \mathrm{MeV}$ alpha partıcles. 
and back and the pulse height was observed as a function of the bias voltage. When irradiated from the front, the point at which the pulse height saturates gives an approximate measure of the active region depth as a function of voltage. When irradiated from the back, the point at which the pulse height abruptly increases also gives an indication of the active region depth. The results for a $45 \mu \mathrm{m}$ thick detector and a 100 $\mu \mathrm{m}$ thick detector are shown in Fig. 4. As can be seen, the active region clearly requires considerable applied voltage before extending completely across either device. Comparison of the voltages at which the pulse height begins to increase from back side irradiation indicates that the active region widths demonstrate an almost linear dependence on applied voltage.

Bulk GaAs detectors irradiated with low energy gamma rays ( 22 to $122 \mathrm{keV}$ ) demonstrated resolutions between approximately 20 and 30\% FWHM [17-20]. The poor resolution is attributed mainly to poor carrier collection and position dependent charge collection. Pulses from higher energy gamma rays $(662 \mathrm{keV})$ were observed only as a continuum of counts and distinct photopeaks did not appear.

Measurements with pulsed X-rays indicated that the charge carriers suffered short lifetimes in the range of only a few nanoseconds [17]. Unfortunately, the short carrier lifetimes translate into low carrier collection efficiency for thick detectors. The current response to pulsed $\mathrm{X}$-rays showed two time-resolved components. Most of the charge was collected over a very short period of time (ns), whereas at low voltages a tail region was observed to extend beyond $50 \mathrm{~ns}$. The extended tail region may be due to slow hole velocities and carrier detrapping of both electrons and holes. $I-V$ measurements demonstrated space charge limited forward currents with increasing turn on voltages with detector thickness [17]. The indication again is the presence of a high density of carrier traps that limit the current until a trap filled limit voltage $\left(V_{\mathrm{TFL}}\right)$ is reached [36]. The above measurements all indicate that the electrical properties of undoped SI LEC bulk GaAs suffer considerably from the presence of a high density of carrier trapping centers.

\section{Proposed electric field models}

Although undoped SI LEC bulk GaAs material has a very low free carrier concentration $\left(10^{6}-10^{7}\right.$ per $\mathrm{cm}^{3}$ ), the reported bulk GaAs devices behaved more as extrinsic detectors rather than intrinsic detectors. The low free carrier concentration and high resistivity of undoped SI LEC bulk GaAs are achieved by the compensation of residual shallow acceptor impurities (usually carbon) with the native deep donor defect denoted EL2 [37,38]. We believe that the detector active region width is determined by the concentration of ionized deep dopants and shallow dopants [17,39-41]. A simple model in which one type of shallow acceptor, deep acceptor, shallow donor, and deep donor are present in the band gap gives

$$
\begin{aligned}
-\frac{\partial^{2} V}{\partial x^{2}}=\frac{\partial \mathscr{E}}{\partial x}= & \frac{q}{\epsilon_{\mathrm{s}}}\left(N_{\mathrm{d}}^{+}(x)-N_{\mathrm{d}}^{-}(x)+N_{\mathrm{DD}}^{+}(x)\right. \\
& \left.-N_{\mathrm{AA}}^{-}(x)-n(x)+p(x)\right),
\end{aligned}
$$

where $N_{\mathrm{DD}}^{+}$is the ionized deep donor concentration, $N_{\mathrm{AA}}^{-}$is the ionized deep acceptor concentration, $N_{\mathrm{d}}^{+}$is the shallow donor concentration, $N_{\mathrm{a}}^{-}$is the shallow acceptor concentration, $n$ is the electron concentration, $p$ is the hole concentration, $q$ is the charge of an electron, and $\epsilon_{\mathrm{s}}$ is the dielectric constant of the material. GaAs material vendors generally report measured shallow acceptor concentrations between $10^{14} / \mathrm{cm}^{3}$ and $10^{15} / \mathrm{cm}^{3}$ and deep donor EL2 concentrations on the order of $10^{16} / \mathrm{cm}^{3}$. Independent measurements utilizing an optical transmission technique $[42,43]$ indicated that the material used for our detectors had acceptor concentrations on the order $10^{15} / \mathrm{cm}^{3}$ and deep donor EL2 concentrations on the order of $10^{16} / \mathrm{cm}^{3}$ [44]. At room temperature, the shallow dopants are assumed to be fully ionized such that $N_{\mathrm{a}}^{-}=N_{\mathrm{a}}$ and $N_{\mathrm{d}}^{+}=N_{\mathrm{d}}$. Several electric field models involving the doping concentrations are discussed in the following sections. The calculated electric fields are all shown in absolute value. The detector configuration modeled in all cases was a Schottky barrier diode with a diffused ohmic contact. The numerical solutions to the electric field distributions were used to estimate the electron and hole velocities as a function of the detector depth. The Bragg ionization distribution was used to estimate the alpha particle energy deposition per unit path length [34]. Carrier trapping was also taken into account for the calculated pulse height of the total charge collected. In all cases discussed, the electron and hole lifetimes were estimated to be $10 \mathrm{~ns}$ [23].

\subsection{The intrinsic case $\left(N_{\mathrm{d}}=N_{\mathrm{d}}\right.$ and $\left.N_{\mathrm{AA}}=N_{\mathrm{DD}}\right)$}

In the case where the material is intrinsic or the case where the impurities are perfectly balanced, the net space charge in the detector volume is simply zero. The solution to Eq. (1) for a $100 \mu \mathrm{m}$ detector indicates that a constant electric field that increases with bias voltage should be expected (Fig. 5). The electric field extends the full length of the detector under all reverse bias conditions. Therefore the active region of the detector extends from the Schottky contact to the ohmic contact. The expected pulse height responses to $3.1,5.5$, and $8.78 \mathrm{MeV}$ alpha particles for a $100 \mu \mathrm{m}$ detector when irradiated from the front or back can be seen in Fig. 6. The pulse height is seen to increase 


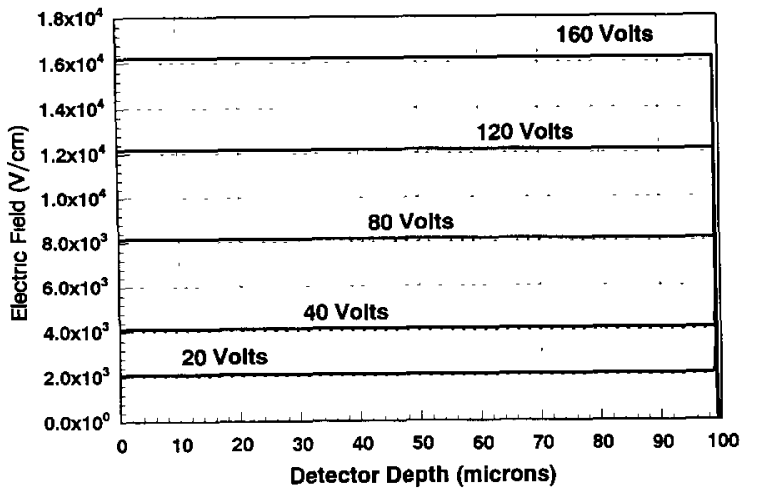

Fig. 5. Expected electric field distributions at different bias voltages for a $100 \mu \mathrm{m}$ Schottky diode detector where the material is intrinsic or the impurities are in perfect balance. The detector depth refers to the distance from the reverse biased Schottky contact.

immediately upon the application of bias voltage if irradiated from the front (Schottky contact). The pulse height should increase immediately upon the application of bias voltage if irradiated from the back (ohmic contact), a case never observed experimentally. Therefore, the intrinsic model fails to account for the experimentally observed pulse height characteristics and the bulk GaAs diodes cannot be treated as intrinsic or perfectly compensated material.

\subsection{The case in which $N_{\mathrm{a}} \gg N_{\mathrm{d}}$ and $N_{\mathrm{DD}} \gg N_{\mathrm{AA}}$}

The measured energy level of the native defect deep donor EL2 is $0.8 \mathrm{eV}$ below the conduction band edge $[37,38]$. Assuming the Fermi level to be near midgap, the deep donors are not fully ionized and the ionized concentration of donors is

$N_{\mathrm{DD}}^{+}=N_{\mathrm{DD}}\left[1-\frac{1}{1+\frac{1}{2} \exp \left(\frac{E_{\mathrm{DD}}-E_{\mathrm{fn}}}{k T}\right)}\right]$,

where $N_{\mathrm{DD}}$ is the deep donor concentration, $E_{\mathrm{DD}}$ is the deep donor energy, and $E_{\mathrm{fn}}$ is the n quasi-Fermi energy $[45,46]$. The application of reverse voltage causes the conduction band to bend such that the energy difference between the deep donor energy level and the quasi-Fermi energy level becomes larger (Fig. 7). The result is further ionization of the deep donor level. Thus, the ionization of the deep donors present in the bulk crystal becomes a function of the bias voltage.

The numerical solution of Eq. (1) with an applied reverse bias results in a net ionization distribution $\left(\left|N_{\mathrm{DD}}^{+}-N_{\mathrm{a}}^{-}\right|\right)$and an electric field distribution $(E)$ with two distinctively different regions (Fig. 8) $[17,35,39,40]$. The solution indicates that the Schottky barrier potential causes full ionization of the deep donors at the Schottky contact. With the application of increasing reverse bias, the region of complete deep level ionization extends further into the detector bulk. Consequently, the non-uniform ionization distribution creates a two zone electric field distribution across the detectors. From Fig. 8, the electric field distribution near the Schottky contact decreases rapidly with a slope approximately equal to $q N_{\mathrm{DD}} / \epsilon_{\mathrm{s}}$. At the end of this region, the deep donors are no longer fully ionized and the electric field decreases less rapidly. The application of voltage causes the electric field to increase across the detector; however, the two zone electric field distribution is still present. The electric field decreases to zero before reaching the ohmic contact. Thus, the model indicates that deep levels in semi-insulating GaAs can severely alter the electric field distribution in the detectors.

Although the model serves to demonstrate the perturbation of the electric field in the presence of deep donors, it fails to account for the observed pulse height characteristics. The high field region is dominated almost entirely by the total concentration of deep donor levels, and consequently, only a few volts are represented by the low field region. Under such conditions, the necessary bias voltage to reach full depletion is much greater than observed in practice. It becomes obvious that the net density of space charge in the active region must be less than that represented by the total deep donor concentration if the active region is to extend fully across the detectors as has been experimentally observed.

\subsection{The case in which $N_{\mathrm{a}} \gg N_{\mathrm{d}}$ and $N_{\mathrm{DD}}>N_{\mathrm{AA}}$}

The deep donor level EL2 has been shown to often be accompanied by a similar deep acceptor level with

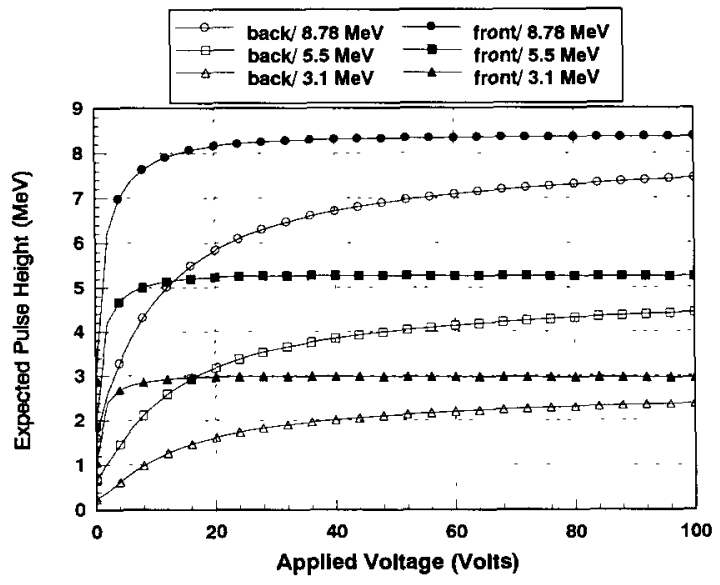

Fig. 6. Expected pulse height response to $3.1,5.5$, and 8.78 $\mathrm{MeV}$ alpha particles as a function of bias voltage for a 100 $\mu \mathrm{m}$ intrinsic GaAs detector. Notice that pulses should be observed both from front and back irradiation with the applcation of only a few volts. 


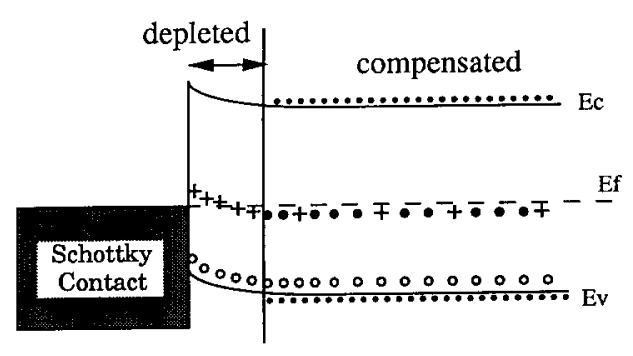

(a)

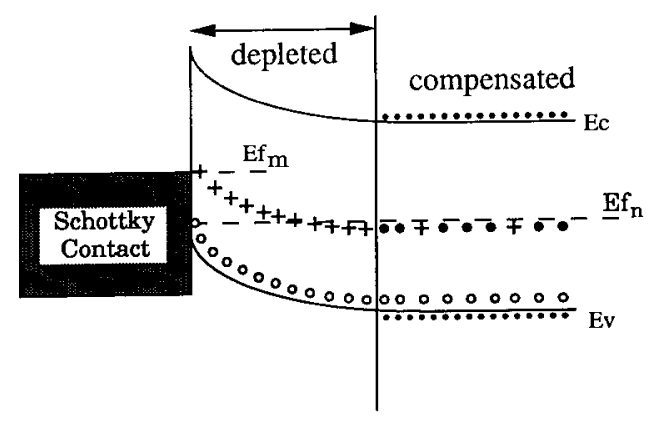

(b)

Fig. 7. Band diagrams of a Schottky contact diode with shallow acceptors and compensating deep donors in the band gap. With no reverse voltage applied (a), the contact potential causes high ionization of the deep donors such that a considerable space charge region appears near the Schottky contact. With the application of reverse voltage (b), the region of high deep donor ionization extends further into the material thus increasing the space charge region. The neutral (or filled) deep donors are represented by $\bullet$, the ionized deep donors (positive charge) are represented by + , and the ionized shallow acceptors (negative charge) are represented by $O$

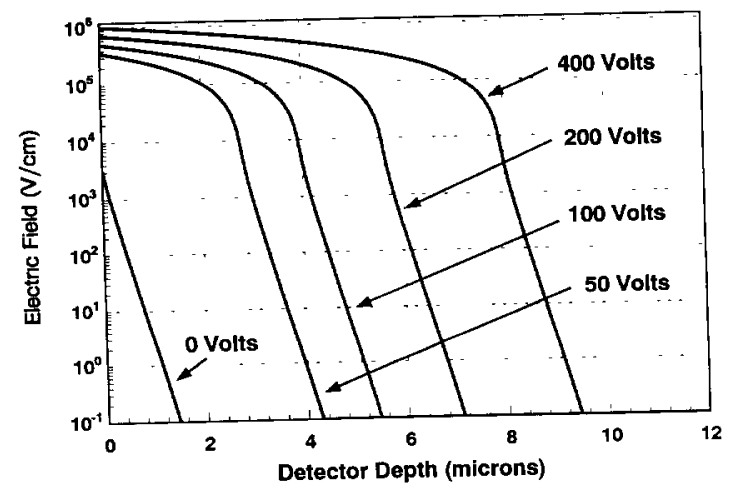

Fig. 8. Expected electric field distributions at different bias voltages for a Schottky diode detector where the material has a high concentration of deep donors. The concentrations used for the calculation were $N_{\mathrm{a}}^{-}=10^{15} / \mathrm{cm}^{3}$ and $N_{\mathrm{DD}}=$ $10^{16} / \mathrm{cm}^{3}$. The detector depth refers to the distance from the reverse biased Schottky contact

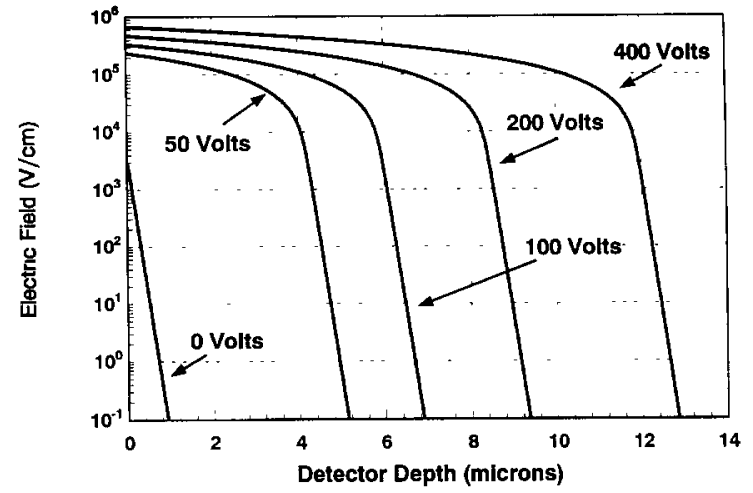

F1g. 9. Expected electric field distributions at different bias voltages for a Schottky diode detector where the material has a high concentration of deep donors and deep acceptors. The concentrations used for the calculation were $N_{\mathrm{a}}^{-}=10^{15} / \mathrm{cm}^{3}$, $N_{\mathrm{AA}}=5 \times 10^{15} / \mathrm{cm}^{3}$, and $N_{\mathrm{DD}}=10^{16} / \mathrm{cm}^{3}$. The detector depth refers to the distance from the reverse biased Schottky contact.

energies of 0.078 and $0.2 \mathrm{eV}$ above the valence band edge $[47,48]$. The deep acceptor is thought to be associated with the $\mathrm{Ga}$ antisite $\left(\mathrm{Ga}_{\mathrm{As}}\right)$. If the concentration of $\mathrm{Ga}_{\mathrm{As}}$ is significantly large by comparison to the EL2 concentration, then the space charge in the active region will reflect the net concentration of $\mid N_{\text {DD }}^{+}-$ $N_{\text {AA }}^{-} \mid$. The ionization of the deep acceptors is represented by

$N_{\mathrm{AA}}^{-}=N_{\mathrm{AA}}\left[1-\frac{1}{1+\frac{1}{4} \exp \left(\frac{E_{\mathrm{fp}}-E_{\mathrm{AA}}}{k T}\right)}\right]$,

where $E_{\mathrm{AA}}$ is the deep acceptor energy and $E_{\mathrm{fp}}$ is the p quasi-Fermi energy. Fig. 9 shows the numerical result of the condition where $N_{\mathrm{DD}}=10^{16} / \mathrm{cm}^{3}, N_{\mathrm{AA}}=5 \times$ $10^{15} / \mathrm{cm}^{3}$, and $N_{\mathrm{a}}=10^{15} / \mathrm{cm}^{3}$. The presence of more positive charge in the detector active region causes the net space charge to decrease such that the electric field extends further into the detector bulk than the case in which deep acceptors are insignificant. However, it again fails to explain the experimentally observed results since the model indicates that voltages much higher than those observed are necessary to extend the active region across the detector.

\subsection{The case in which $N_{\mathrm{DD}}=N_{\mathrm{AA}}$}

If the total concentration of deep acceptors is equal to the total concentration of deep donors (highly improbable), then the expected electric field is dominated almost entirely by the concentration of residual shallow dopants. Therefore, the electric field will extend into the detector bulk much further as restricted by the 


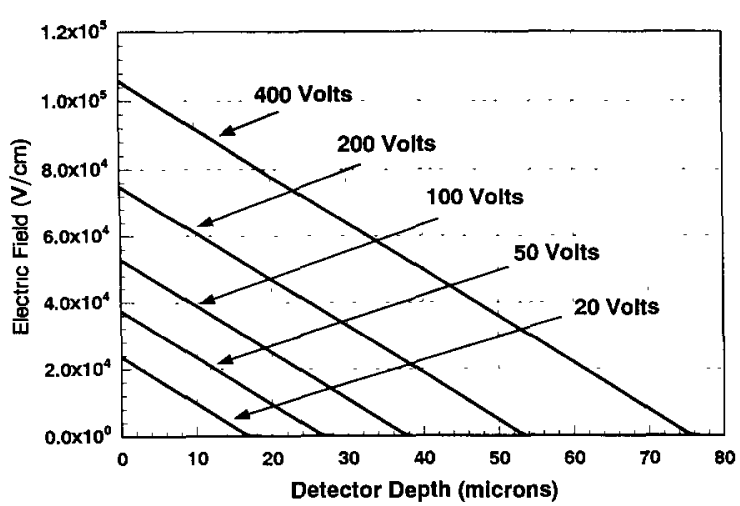

Fig. 10. Expected electric field distributions at different bias voltages for a $100 \mu \mathrm{m}$ Schottky diode detector where the concentration of deep donors and deep acceptors are equal. The net concentration of shallow dopants used for the calculation was $\left|N_{\mathrm{d}}^{-}-N_{\mathrm{d}}^{+}\right|=10^{14} / \mathrm{cm}^{3}$. The detector depth refers to the distance from the reverse biased Schottky contact.

shallow dopant concentration. As shown in Fig. 10, the electric field extends much further into the detector depth and decreases with a slope defined by the net shallow dopant concentrations. The expected alpha particle pulse height curves for a $45 \mu \mathrm{m}$ detector and a $100 \mu \mathrm{m}$ detector are shown in Fig. 11. Notice that the calculated response for the $45 \mu \mathrm{m}$ detector (Fig. 11a) provides a closer match to the measured result. However, the calculated response for the $100 \mu \mathrm{m}$ detector (Fig. 11b) is markedly different from the measured response. The model indicates that the electric field should demonstrate a growing active region depth that is proportional to $\sqrt{V}$, a case never found experimentally. The model indicates that full active region extension for a $100 \mu \mathrm{m}$ detector should require over four times the voltage than the $45 \mu \mathrm{m}$ detector requires. As shown in Fig. 4, the voltage necessary to extend the active region across the $100 \mu \mathrm{m}$ detector was only twice that of the $45 \mu \mathrm{m}$ detector, which demonstrates a linear dependence with applied voltage rather than a $\sqrt{V}$ dependence.

From the experimental data, it becomes obvious that the detectors do not follow the behavior expected from intrinsic or perfectly compensated material. Simple models invoking the ionization of deep levels fail to adequately explain the observed pulse height characteristics. In the intrinsic model the active region extends fully across the device with only a few volts, whereas in the ionized deep level model the active region requires extensive voltage to extend across the device. We therefore conclude that a more complex model is required to explain the observed behavior.

\subsection{The case in which the deep donors experience field enhanced capture and emission}

It has been demonstrated that the deep level EL2 undergoes dramatic changes with carrier capture cross sections and emission rates at increasing electric field strengths beneath the Schottky contact [49-52]. The electron emission rate from the EL2 centers goes through a minimum when the electric field is increased from 0 to $10^{5} \mathrm{~V} / \mathrm{cm}$. Beyond $10^{5} \mathrm{~V} / \mathrm{cm}$, the emission rate increases several orders of magnitude and may be attributed to impact ionization of the deep levels from injected electrons. It has been reported that the measured EL2 concentration appears to decrease with the free electron density and increased leakage current [52]. The electron and hole capture rate for EL2 has been reported to increase several orders of magnitude near a critical electric field $\mathscr{E}_{c}$ of about $10^{4} \mathrm{~V} / \mathrm{cm}$ $[49,50]$. Injected charge through a forward biased ohmic contact can supply the necessary carriers to refill the
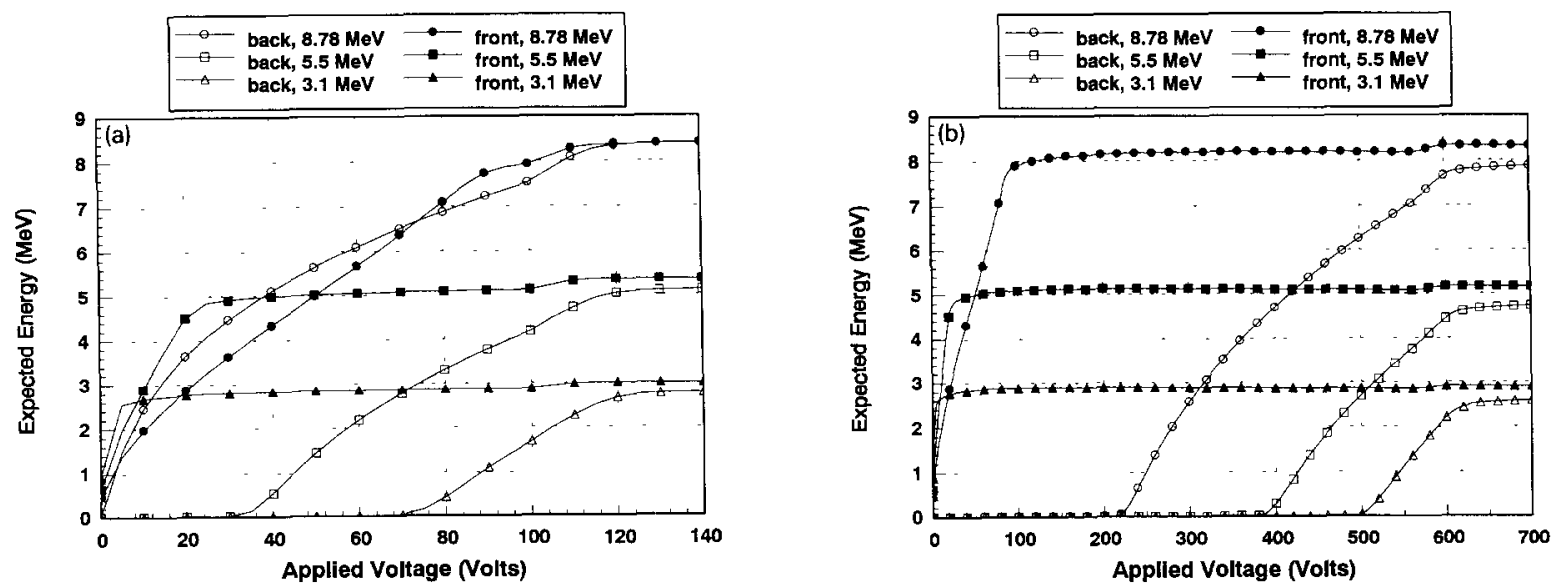

Fig. 11. Expected pulse height responses to 3.1, 5.5, and $8.78 \mathrm{MeV}$ alpha particles as a function of bias voltage for (a) a $45 \mu \mathrm{m}$ GaAs detector and (b) a $100 \mu \mathrm{m}$ GaAs detector. The conditions for the calculation were $N_{\mathrm{DD}}=N_{\mathrm{AA}}$ and $\left|N_{\mathrm{d}}^{-}-N_{\mathrm{d}}^{+}\right|$ $=10^{14} / \mathrm{cm}^{3}$. 
deep donor centers and produce a neutralization effect [53]. If the electron capture rate is higher than the electron emission rate near $\mathscr{E}_{c}$, then the leakage current through a reverse biased Schottky contact may create a case similar to Au doped $\mathrm{Si}$ where a quasineutral region is formed [54].

The conventional assumption for a reverse biased Schottky junction is that all deep donor levels above the $\mathrm{n}$ quasi-Fermi level will be empty (positive ions) and those remaining below the $\mathrm{n}$ quasi-Fermi level will be filled (neutral). However, the relation is derived for near equilibrium conditions and it becomes clear that the detector is not at equilibrium at high bias voltages. The steady state ionized deep donor concentration can be approximated by [46]

$N_{\mathrm{DD}}^{+} \approx \frac{N_{\mathrm{DD}}}{1+n \sigma_{n} v_{e} / e_{n}}$,

where $\sigma_{n}$ is the deep donor electron capture cross section (in $\mathrm{cm}^{2}$ ) and $e_{n}$ is the deep donor electron emission rate per second. If the thermionic leakage current through the Schottky contact is substantial, then the deep donors will no longer be fully ionized. This case may account for the reported reduction in the measured EL2 concentration with increased leakage current [52].

The room temperature electron capture cross section for EL2 reportedly increases from approximately $10^{-15} \mathrm{~cm}^{2}$ at low electric fields to $10^{-13} \mathrm{~cm}^{2}$ at electric fields near $10^{4} \mathrm{~V} / \mathrm{cm}$ [50]. The room temperature emission rate for EL2 is approximately $0.1 / \mathrm{s}$ at electric fields below $10^{5} \mathrm{~V} / \mathrm{cm}[46,51]$. A common leakage current of $100 \mathrm{nA}$ for a $4 \mathrm{~mm}$ diameter device results in an electron density $(n)$ approximately equal to $5 \times$ $10^{5} / \mathrm{cm}^{3}$ flowing through the depletion region. Assuming saturated carrier velocities $\left(10^{7} \mathrm{~cm} / \mathrm{s}\right)$, Eq. (4) gives $N_{\text {DD }}^{+}=0.95 N_{\text {DD }}$ at low electric fields, thus demonstrating that most of the deep donors are ionized. However, at high electric fields where the electron capture cross section has increased to $10^{-13} \mathrm{~cm}^{2}$, Eq. (4) indicates that the ionized EL2 concentration reduces to $0.17 N_{\mathrm{DD}}$. Therefore, the ionized concentration of EL2 deep donors is reduced to the same order of magnitude as the ionized shallow carbon acceptor concentration. The condition supports the interesting possibility that the electric field enhanced capture of electrons can produce a quasi-neutral region in the device depletion region (or active region). Unfortunately, measured data is unavailable for the electron capture cross section at electric fields between 0 and $10^{4} \mathrm{~V} / \mathrm{cm}$, thus no accurate model exists for the electron capture cross section as a function of increasing electric field.

The effect is modeled here by altering Eq. (2) such that the ionization of the deep levels decreases dramat- ically at electric fields approaching $\mathscr{E}_{\mathrm{c}}$. To account for filling of traps at high electric fields, Eq. (2) is altered such that the ionized concentration of the deep donors is represented by

$$
\begin{aligned}
N_{\mathrm{DD}}^{+}= & N_{\mathrm{DD}}\left[1-\frac{1}{1+\frac{1}{2} \exp \left(\frac{E_{\mathrm{DD}}-E_{\mathrm{fn}}}{k T}\right)}\right] \\
& -f\left(N_{\mathrm{DD}}, \mathscr{E}\right),
\end{aligned}
$$

where $f\left(N_{\mathrm{DD}}, \mathscr{E}\right)$ is a filling function that strongly increases the number of filled EL2 traps as the electric field approaches $\mathscr{E}_{\mathrm{c}}$. Since the actual filling function is unknown, an arbitrary function was selected to represent the rapid filling of the deep donor centers at fields approaching $\mathscr{E}_{\mathrm{c}}$ :

$$
\begin{aligned}
N_{\mathrm{DD}}^{+}= & N_{\mathrm{DD}}\left[1-\frac{1}{1+\frac{1}{2} \exp \left(\frac{E_{\mathrm{DD}}-E_{\mathrm{fn}}}{k T}\right)}\right] \\
& -\left(N_{\mathrm{DD}}-N_{\mathrm{a}}\right)\left(\frac{1}{1+\left(\frac{\mathscr{E}_{\mathrm{c}}}{\mathscr{E}}\right)^{\alpha}}\right),
\end{aligned}
$$

where the term $\left(N_{\mathrm{DD}}-N_{\mathrm{a}}\right)$ ensures that the net space charge in the active region cannot change from a net positive charge to a net negative charge. The value of $\alpha$ determines how strong the dependence of the electron capture cross section on field strength becomes. This function serves only to provide the expected trend in the case that deep donor levels begin to strongly capture electrons at high electric fields. Fig. 12 shows the numerically calculated result from Eqs. (1) and (6)

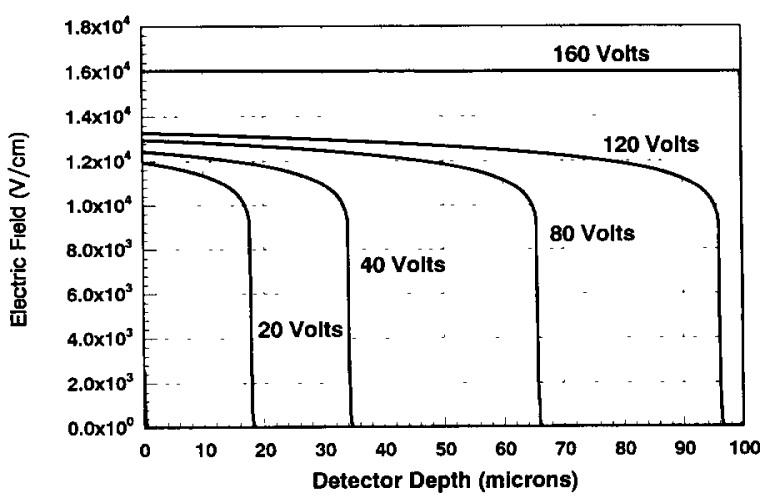

Fig. 12. Expected electric field distributions at different bias voltages for a $100 \mu \mathrm{m}$ Schottky diode detector where deep levels refill at high electric fields. The concentrations used for the calculation were $N_{\mathrm{a}}^{-}=10^{15} / \mathrm{cm}^{3}$ and $N_{\mathrm{DD}}=10^{16} / \mathrm{cm}^{3}$. The value of $\mathscr{E}_{c}$ was $7 \times 10^{3} \mathrm{~V} / \mathrm{cm}$ and $\alpha$ was 15 . The high value of $\alpha$ simulates heavy trap filling as the field strength approaches $10^{4} \mathrm{~V} / \mathrm{cm}$. The detector depth refers to the distance from the reverse biased Schottky contact. 
with $N_{\mathrm{DD}}=10^{16} / \mathrm{cm}^{3}$ and $N_{\mathrm{a}}=10^{15} / \mathrm{cm}^{3} \cdot N_{\mathrm{d}}$ and $N_{\mathrm{AA}}$ are considered negligible. As can be seen, the active region width no longer demonstrates a $\sqrt{V}$ dependence and increases almost linearly with applied voltage. The electric field has two distinct regions. In the first region, the electric field remains practically constant up to an extended distance into the detector bulk. In the second region, the electric field drops abruptly due to the high concentration of ionized deep donors in a lower field region.

The expected pulse height characteristics for alpha particle irradiation indicate a behavior that closely resembles measured results. The expected alpha particle pulse height responses for a $45 \mu \mathrm{m}$ detector and a $100 \mu \mathrm{m}$ detector from the proposed model are shown in Fig. 13. As can be seen, the necessary voltage to extend the active region across the $45 \mu \mathrm{m}$ detector is dramatically less than any of the other models, yet a threshold voltage is still apparent for the onset of pulse height increase from back side irradiation. The calculated pulse height curves in Fig. 13a match well with the measured results in Fig. 4a. The expected response for the $100 \mu \mathrm{m}$ detector (shown in Fig. 13b) also matches well with the experimentally observed results in Fig. 4b. Discrepancies between the front irradiation plateau voltage for the model and the measured results may be due to plasma time recombination effects $[30,55]$.

The observed active region depths are too wide to be accounted for by the known donor and acceptor concentrations and therefore a substantial region of low space charge (or neutral region) must be present. Direct measurements of the potential across similar detectors indicated that a fairly flat electric field region was present followed by a sharp drop in electric field strength [22], which also indicates the formation of a quasi-neutral region. We therefore propose that the field enhancement of the EL2 capture cross section coupled with a low emission rate promotes the formation of a quasi-neutral region at high electric fields. It is not assumed that the EL2 centers remain filled, but that the time averaged effect of the EL2 centers filling and emptying produces a quasi-neutral region at sufficiently high fields. By assuming the production of a quasi-neutral region, our model closely reproduces the alpha particle pulse height curves shown in Fig. 4.

\section{Carrier collection difficulties}

The basic designs presently used for semiconductor detectors require the efficient collection of both electrons and holes if high resolution is to be achieved. For any semiconductor detector, efficient carrier extraction requires that charge carriers have long lifetimes and high saturation velocities. The electron velocity in $\mathrm{GaAs}$ can be high at low electric fields $\left(2 \times 10^{7} \mathrm{~cm} / \mathrm{s}\right.$ at $3 \times 10^{3} \mathrm{~V} / \mathrm{cm}$ ), however the hole velocity is over an order of magnitude lower (near $10^{6} \mathrm{~cm} / \mathrm{s}$ ) due to their lower mobility. Hole saturated velocities are similar to electron saturated velocities (near $10^{7} \mathrm{~cm} / \mathrm{s}$ ) and can be approached by increasing the electric field above $3 \times 10^{4} \mathrm{~V} / \mathrm{cm}$. Unfortunately, the high electron velocity is no longer maintained at these high fields.

The carrier lifetimes observed in GaAs are short, normally on the order of nanoseconds. The short lifetimes are attributed to the high concentrations of various electron and hole traps in the band gap [38,46$48,56-58]$. One set of measurements indicated that hole trapping and detrapping were electric field depen-
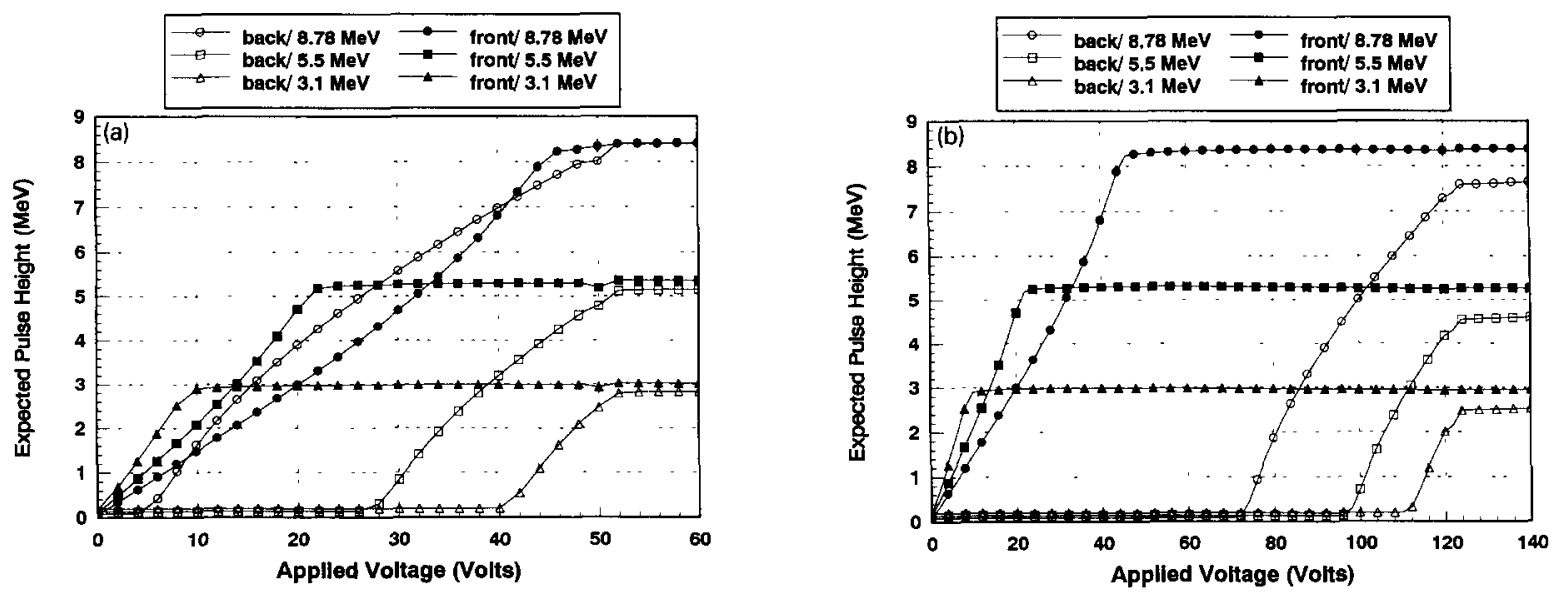

Fig. 13. Expected pulse height response to $3.1,5.5$, and $8.78 \mathrm{MeV}$ alpha particles as a function of bias voltage in which heavy deep donor filling occurs for (a) a $45 \mu \mathrm{m}$ detector and (b) a $100 \mu \mathrm{m}$ detector. The concentrations used for the calculation were $N_{\mathrm{a}}^{-}=10^{15} / \mathrm{cm}^{3}$ and $N_{\mathrm{DD}}=10^{16} / \mathrm{cm}^{3}$. The value of $\mathscr{E}_{\mathrm{c}}$ was $7 \times 10^{3} \mathrm{~V} / \mathrm{cm}$ and $\alpha$ was 15 . The high value of $\alpha$ simulates heavy trap filling as the field strength approaches $10^{4} \mathrm{~V} / \mathrm{cm}$. 
dent and hole lifetimes at high fields were approximately 3-9 ns [17]. An index for charge collection (or pulse height) is referred to as the carrier extraction factor $(\mathrm{CEF})$ :

$\rho=\left(\frac{\tau^{*}}{t}\right)\left(\frac{x}{W}\right)=\left(\frac{\tau^{*} v}{x}\right)\left(\frac{x}{W}\right)=\frac{\tau^{*} v}{W}$,

where $\tau^{*}$ is the carrier lifetime (or mean free drift time), $t$ is the collection time required, $x$ is the distance the carrier must travel, $v$ is the carrier velocity, and $W$ is the detector active region width $[3,35]$. The expected pulse height can be calculated from

$Q=q N_{0}\left(\rho_{\mathrm{e}}\left(1-\mathrm{e}^{-x_{\mathrm{e}} / \rho_{\mathrm{e}} W}\right)+\rho_{\mathrm{h}}\left(1-\mathrm{e}^{-x_{\mathrm{h}} / \rho_{\mathrm{h}} W}\right)\right)$,

where $N_{0}$ is the number of electron-hole pairs produced and $\rho_{\mathrm{e}}$ and $\rho_{\mathrm{h}}$ are the CEF values for electrons and holes, respectively. The mean value and standard deviation of Eq. (8) can give a measure of the expected resolution from gamma ray interactions if the CEFs are known [3,35]. Eqs. (7) and (8) can be used to estimate the degradation in resolution from position dependent charge collection where the resolution is defined as

$\sigma_{q}=\frac{\sqrt{\left\langle Q^{2}\right\rangle-\langle Q\rangle^{2}}}{\langle Q\rangle}=\frac{\sigma_{Q}}{\langle Q\rangle}$.

Fig. 14 shows the expected contribution to the energy resolution from this effect as a function of the electron and hole CEFs where the percent resolution is defined as $100 \sigma_{q}$. Assuming saturated carrier velocities, a $1 \mathrm{~mm}$ thick detector with electron and hole lifetimes of $5 \mathrm{~ns}$

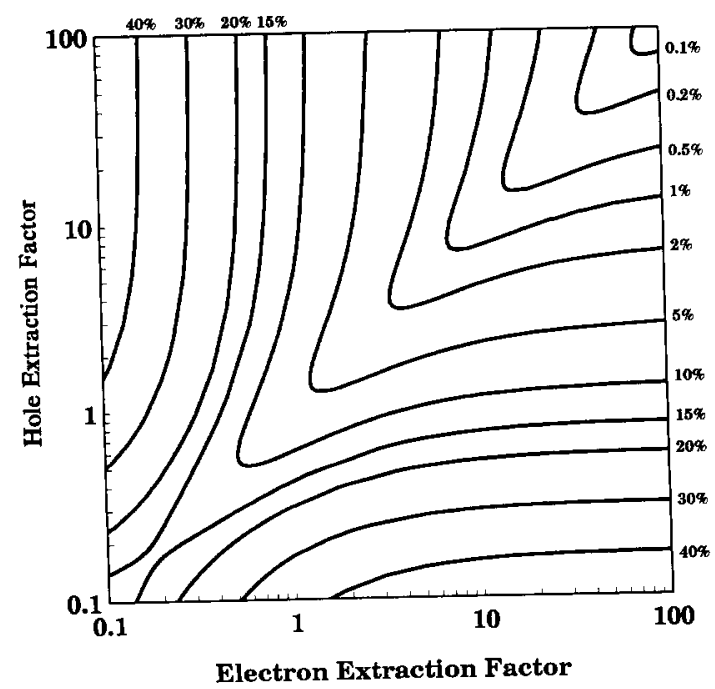

Fig. 14. The detector percent energy resolution, when defined as $100 \sigma_{Q} /\langle Q\rangle$ (or $100 \sigma_{q}$ ) is shown as a function of the carrier extraction factors. Note that, for a Gaussian peak, the conventional definition of resolution is $\mathrm{FWHM} /\langle Q\rangle$, or 2.35 times the values shown. would have CEF values approximately equal to 0.5 , corresponding to an expected resolution of $10.4 \%$. The differential pulse height distribution is typically skewed to one side with high carrier trapping, however in the case that the differential pulse height spectrum is Gaussian the resolution would be approximately $25 \%$ at FWHM. If the carrier lifetimes are unequal such that the electron lifetime is $10 \mathrm{~ns}$ and the hole lifetime is $5 \mathrm{~ns}$, the resolution would broaden to $12.5 \%$ (or for a Gaussian peak, 30\% at FWHM). From Eq. (8), it is also apparent that the pulse height (or charge collected) decreases as the CEF values decrease. Therefore, the signal to noise ratio decreases and the resolution broadens further due to carrier statistics and/or electronic noise. A GaAs detector with resolution within $1 \%$ would require increasing the carrier lifetimes above $100 \mathrm{~ns}$ for a $1 \mathrm{~mm}$ thick detector and above $1 \mu \mathrm{s}$ for a $1 \mathrm{~cm}$ thick detector.

\section{Discussion}

Some difficulties imposed by the presence of deep levels in a compensated semiconductor have been outlined. Although native defect deep level compensation of $\mathrm{GaAs}$ provides a convenient method by which the resistivity of the material is tailored, its presence causes extreme difficulties for high resolution gamma ray detectors. Deep level compensation of background impurities provides adequate material for the production of VLSI devices since the semi-insulating (SI) GaAs material is generally used as a substrate or altered by implantation. However, SI GaAs compensated with deep levels does not meet the stringent material requirements necessary for a high resolution room temperature gamma ray detector.

The first major problem with SI LEC compensated $\mathrm{GaAs}$ is the electric field distribution, in which the field is altered due to ionization of deep levels with reverse bias. Although the electric field may in fact be flat across a portion of the detector, the required voltage for depletion of only a few $\mathrm{mm}$ thickness goes well beyond that necessary for HPGe detectors and $\mathrm{Si}(\mathrm{Li})$ detectors of $\mathrm{cm}$ in thickness. The efficiency of GaAs detectors of such small size cannot compete with present day HPGe detectors.

The second major problem is the fact that the presence of deep levels or traps in a direct band gap semiconductor dramatically reduce the carrier lifetimes. In the presence of severely compensated material, the carrier extraction factors will ultimately be reduced to levels inconsistent with that necessary for high resolution spectroscopy. Therefore, even if the detectors were depleted beyond several $\mathrm{cm}$, the extremely short carrier lifetimes would result in CEFs too small for a useful spectrometer. 
Since it is speculated that the presence of deep levels severely alters the performance of bulk SI LEC $\mathrm{GaAs}$, it seems evident that a significant reduction in the deep level concentrations must occur before a viable room temperature bulk GaAs gamma ray detector will be realized. Previous LPE GaAs detectors clearly demonstrated that GaAs of excellent purity can be produced. The GaAs material described in these previous works was of the highest purity available and was not compensated with deep level impurities or native defects. Therefore, it seems only reasonable that efforts towards the production of bulk GaAs detectors in the future should be directed towards the improvement of the bulk material itself. Present day bulk GaAs growth is generally performed with arsenic and gallium starting material that is only 7 nines in purity [59]. Higher purity ( 8 nines) starting material is available and should be used as a first step towards cleaner bulk crystals. Growth rates can be altered to allow for higher stoichiometric control. Presently, GaAs is grown arsenic rich to purposely create EL2 deep donors in high concentration. Efforts should instead be made to approach a true one-to-one stoichiometric mix of $\mathrm{Ga}$ and As.

Bulk GaAs has been demonstrated as an alternative alpha particle, beta particle, and conversion electron spectrometer [14,17-19], however the resolution and room temperature operability of bulk GaAs detectors show no practical advantage over present day Si based detectors. Particle detectors fabricated from LPE GaAs demonstrated results that closely approached the performance of Si. However, the cost of LPE GaAs layers of thicknesses required for particle spectroscopy offers no clear advantage over zone refined Si. Gamma rays at erergies of 60 and $122 \mathrm{keV}$ have been measured with very thin bulk GaAs crystals with resolutions at FWHM approaching 20-30\% [17-20] and some improvement has been reportedly observed from heat treated bulk GaAs material [22]. However, the advantage of room temperature operation at the expense of such poor resolution hardly competes with present day (cooled) $\mathrm{Si}(\mathrm{Li})$ detectors. Epitaxially grown $\mathrm{GaAs}$ detectors with high resolution will likely continue to be limited to small dimensions impractical for high energy gamma ray spectroscopy. We conclude that large bulk $\mathrm{GaAs}$ room temperature gamma ray spectrometers will become a reality only if improved bulk crystal growth processes are researched and implemented such that high purity material is produced with significantly reduced impurity and native defect trap concentrations.

\section{Acknowledgements}

The authors express their gratitude to Dr. Millard G. Mier of Wright Patterson Air Force Base for pro- viding measurements of the neutral and total EL2 densities of the bulk LEC GaAs reported in this work. We also express gratitude towards Dr. David C. Look at Wright State University for helpful discussions concerning the electrical properties of GaAs.

This project has been funded in part by Los Alamos National Laboratory subcontract 9-XGO-K7910-1.

\section{References}

[1] S.P. Swierkowski and G.A. Armantrout, IEEE Trans. Nucl. Sci. NS-22 (1975) 205.

[2] G.A. Armantrout, S.P. Swierkowski, J.W. Sherohman and J.H. Yee, IEEE Trans. Nucl. Sci. NS-24 (1977) 121.

[3] G.F. Knoll and D.S. McGregor, MRS Proc. 302 (1993) p. 3.

[4] W.R. Harding, C. Hilsum, M.E. Moncaster, D.C. Northrop and O. Simpson, Nature 187 (1960) 405.

[5] T. Kobayashi and S. Takayanagi, Nucl. Instr. and Meth. 44 (1966) 145

[6] J.E. Eberhardt, R.D. Ryan and A.J. Tavendale, Appl. Phys. Lett. 17 (1970) 427.

[7] J.E. Eberhardt, R.D. Ryan and A.J. Tavendale, Nucl. Instr. and Meth. 94 (1971) 463.

[8] T. Kobayashi and T. Sugita, Nucl. Instr. and Meth. 98 (1972) 179.

[9] K. Hesse, W. Gramann and D. Höppner, Nucl. Instr. and Meth. 101 (1972) 39.

[10] P.E. Gibbons and J.H. Howes, IEEE Trans. Nucl. Sci. NS-19 (1972) 353.

[11] T. Kobayashi, T. Sugita, M. Koyama and S. Takayanagi, ibid., p. 324.

[12] T. Kobayashi, T. Sugita, S. Takayanagi, M. Iio and Y. Sasaki, IEEE Trans. Nucl. Sci. NS-20 (1973) 310.

[13] T. Kobayashi, I. Kuru, A. Hojo and T. Sugita, IEEE Trans. Nucl. Sci. NS-23 (1976) 97.

[14] R. Bertin, S. D'Auria, C. Del Papa, F. Fiori, B. Lisowski, V. O'Shea, P.G. Pelfer, K. Smith and A. Zichichi, Nucl. Instr. and Meth. A 294 (1990) 211.

[15] T.J. Sumner, S.M. Grant, A. Bewick, J.P. Li, N.J.C. Spooner, K. Smith and S.P. Beaumont, Proc. SPIE 1549 (1991) 256.

[16] C.M. Buttar, F.H. Combley, I. Dawson, M. Dogru, M. Harrison, G. Hill, Y. Hou and P. Houston, Nucl. Instr. and Meth. A 310 (1991) 208.

[17] D.S. McGregor, G.F. Knoll, Y. Eisen and R. Brake, IEEE Trans. Nucl. Sci. NS-39 (1992) 1226.

[18] D.S. McGregor, G.F. Knoll, Y. Eisen and R. Brake, Nucl. Instr. and Meth. A 322 (1992) 487.

[19] K.W. Benz, R. Irsigler, J. Ludwig, J. Rosenzweig, K. Runge, F. Schäfer, J. Schneider and M. Webel, Nucl. Instr. and Meth. A 322 (1992) 493.

[20] T.J. Sumner, S.M Grant, A. Bewick, J.P. Li, K. Smith and S.P. Beaumont, ibid., p. 514.

[21] N.J.C. Spooner, A. Bewick, J.J. Quenby, P.F. Smith and J.D. Lewin, in: GaAs Detectors and Electronics for High-Energy Physics, eds. C. del Papa, P.G. Pelfer and K. Smith (World Scientific, Singapore, 1992) p. 156.

[22] K. Berwick, M.R. Brozel, C.M. Buttar, M. Cowperthwaite and Y. Hou, MRS Proc. 302 (1993) p. 363. 
[23] E. Sakai, Nucl. Instr. and Meth. 196 (1982) 121.

[24] S.M. Sze, Physics of Semiconductor Devices, 2nd ed. (Wiley, New York, 1981).

[25] M. Shur, GaAs Devices and Circuits (Plenum, New York, 1987).

[26] R.N. Hall, Proc. IEE 106B (Sup. 17) (1959) 923.

[27] J.W. Mayer, Nucleonics 20 (1962) 60.

[28] N. Holonyak, Jr. and S.F. Bevacqua, Appl. Phys. Lett. 2 (1963) 71.

[29] D.C. Northrop, P.R. Thornton and K.E. Trezise, SolidState Electron. 7 (1964) 17.

[30] G. Dearnaley and D.C. Northrop, Semiconductor Counters for Nuclear Radiations (E.\&F.N. Spon, London, 1964).

[31] M. Kaminska, J.M. Parsey, J. Lagowski and H.C. Gatos, Appl. Phys. Lett. 41 (1982) 989.

[32] D.A. Johnson, Ph.D. dissertation, Arizona State University (1989).

[33] N. Derhacobian and N.M. Haegel, Phys. Rev. B 44 (1991) 12754.

[34] J.F. Ziegler and J.P. Biersack, TRIM-90, Version 90.05 (1990).

[35] D.S. McGregor, Ph.D. dissertation, University of Michigan (1993).

[36] M.A. Lampert and P. Mark, Current Injection in Solids (Academic Press, New York, 1970).

[37] R.K. Willardson and A.C. Beer, Semiconductors and Semimetals, vol. 20 (Academic Press, Orlando, 1984).

[38] J.C. Bourgoin, H.J. von Bardeleben and D. Stievenard, J. Appl. Phys. 64 (1988) R65.

[39] A.G. Milnes, Deep Impurities in Semiconductors (Wiley, New York, 1973).

[40] S.M. Ryvkin, L.L. Makovsky, N.B. Strokan, V.P. Subashieva and A.Kh. Khusainov, IEEE Trans. Nucl. Sci. NS-15 (1968) 226.

[41] J.V. DiLorenzo and D.D. Khandelwal, GaAs FET Principles and Technology (Artech House, Dedham, 1982).
[42] S.K. Brierley and D.S. Lehr, Appl. Phys. Lett. 55 (1989) 2426.

[43] M.G. Mier, D.C. Look, D.C. Walters and D.L. Beasley, Solid-State Electron. 35 (1992) 319.

[44] M.G. Mier, private communication, Wright Patterson Air Force Base (1992).

[45] J.P. McKelvey, Solid State and Semiconductor Physics (Kreiger, Malabar, 1986).

[46] D.C. Look, Electrical Characterization of GaAs Materials and Devices (Wiley, New York, 1989).

[47] K.R. Elliot, D.E. Holmes., R.T. Chen and C.G. Kırkpatrick, Appl. Phys. Lett. 40 (1982) 898.

[48] T. Figielski, Appl. Phys. A 35 (1984) 255.

[49] V.Ya. Prinz and B.A. Bobylev, Sov. Phys. Semicond. 14 (1980) 1097.

[50] V.Ya. Prinz and S.N. Rechkunov, Phys. Status Solidi B 118 (1983) 159.

[51] S. Makram-Ebeid, MRS Proc. 2 (1981) p. 495.

[52] S.T. Pantelides, Deep Centers in Semiconductors, 2nd ed. (Gordon and Breach, New York, 1992).

[53] J.C. Adams, C.D. Capps, R.A. Falk and S.G. Ferrier, Appl. Phys. Lett. 63 (1993) 633.

[54] P.T. Panousis, R.H. Krambeck and W.C. Johnson, Appl. Phys. Lett. 15 (1969) 79.

[55] A.A. Quaranta, A. Taroni and G. Zanarini, IEEE Trans Nucl. Sci. NS-15 (1968) 373.

[56] G.M. Martin, A. Mitonneau and A. Mircea, Electron. Lett. 13 (1977) 191.

[57] A Mitonneau, G.M. Martin and A. Mircea, Electron. Lett. 13 (1977) 666.

[58] G.M. Martin, J.P. Farges, G. Jacob, J.P. Hallais and G. Poiblaud, J. Appl. Phys. 51 (1980) 2840.

[59] R.M. Ware, private communication, Ware Technical Services (1993). 